\title{
DIGITAL DEPENDENCE OF HIGHER EDUCATION EVALUATION: CHALLENGES AND RESOLUTIONS
}

\begin{abstract}
Jiali Yao ${ }^{1}$
Abstract. The Digital dependence in this study is not a dependency on the Internet in traditional contexts. The digital dependence of higher education evaluation has the following manifestations: The widespread penetration of "digit" exists everywhere in higher education evaluation; "Digit" takes up the priority within the coordinate system of higher education evaluation; Irrational "digit" becomes disciplinary force by alienation in higher education evaluation. Its roots in the fact that it derives from the long-term restriction by the evaluation views on rationalism under the control of technological rationality, the promotion of simplifying perception of the higher education field, and the resource black hole and benefit inducement that "digit" can be manifested. Therefore, the key to eliminate the digital dependence in higher education evaluation and breaking the utilitarian thinking should include the following three aspects: returning the meaning of digital criterion in higher education evaluation to be genuine, clarifying the reasonable boundaries of the digital application in the evaluation of higher education, and optimizing the structure of the higher education evaluation system.
\end{abstract}

Keywords: Higher education; education evaluation; digital criterion; digital dependence.

\section{Introduction}

Generally, higher education evaluation is a systematic project that judges and evaluates the operating status, nature, and effectiveness of higher education based on specific principles, standards, and a series of methods and measures. For a long time, digital has become the dominant tool in higher education evaluation by virtue of its "intuitive and accurate", "convenient and efficient" characteristics, and has been taken for granted, which led to the digital application in higher education evaluation simplified and to the organization operation of higher education all-round hijacked. Regardless of external evaluation or internal evaluation, higher education evaluation shows distinct characteristics of digital dependence.

In fact, as a kind of evaluative tool or symbol, it is beyond reproach for digit to appraise subject and object's identification and dependence on it. But when digital tools are admired to extreme levels and become disciplinary character, the existence of humans in the field of higher education is dispelled in the abstract "collection of digit", and the richness and depth of its functions are obscured. What's more, disorderly competition and academic ecological imbalance between and within universities are intensifying. From this, the "Overall Plan for Deepening Educational Evaluation Reform in the New Era" issued by the Central Committee of the Communist Party of China and the State Council clearly proposes to "dilute number of papers included, citation rate, number of awards and other quantitative indicators", and provides institutional and policy orientation for governing the "digital dependence" in higher education evaluation.[1] In order to effectively implement the above spirit, this article intends to unscramble and analyze the "digital dependence" of higher education evaluation, attempting to propose dispelling ways.

\footnotetext{
${ }^{1}$ Faculty of Education, Southwest University, Chongqing,China, 400715;

e-mail: 9406874@qq.com
} 


\section{Actual representation of the digital dependence in higher education evaluation}

\subsection{Digital abuse: The widespread penetration of "digit" exists everywhere in higher education evaluation}

Regardless of universities in macro-level, colleges in meso-level, or teacher and student behaviors in micro-level, to a large extent, they are subtly reduced to numbers, deriving an invisible digital pressure transmission chain, and digital labeling permeates every corner of higher education evaluation from top to bottom. It should be noted that these numbers are further integrated into a complete digital system, constructing an indestructible digital collection, and diffracting at everywhere of higher education evaluation. The wide range of frequent using numbers to measure the quality of higher education has caused complex and profound higher education activities to be reduced to a collection of refined numbers. "The ideals, beliefs, spirit, realm, responsibility, purpose, integrity, and other qualities that universities rely on are squeezed out of place by this digital wave. To a great extent, universities have fallen into the trap of digitization. It seems that its technological, instrumental, and vulgarized trend is hard to reverse."[2]

\subsection{Digital priority: "Digit" takes up the priority within the coordinate system of higher education evaluation}

Although the rational use of multiple scales to comprehensively consider the quality of higher education is the basic criterion and general consensus of evaluation, it is obvious that the actual higher education evaluation prefers digital scales in the selections of evaluation methods. Specifically, the priority of quantitative evaluation methods based on "numbers" is mainly manifested as follows: First, the "digital" scale is decisive. Higher education evaluation mostly makes judgments based on digital scales, and intentionally or unintentionally ignores other judgment scales besides numbers. The digital scale is not only the prerequisite and basis for the evaluation of higher education, but also the standard that the evaluation of higher education abides by. As a result, both quantifiable and non-quantifiable evaluation objects are measured by numbers, such as student ideological and moral scoring, teacher ethics grading, etc. Second, the "digital" scale cannot be flexible. In order to ensure the so-called "objectivity" and "fairness" of evaluation, estimators often implement a one-size-fits-all evaluation based on accurate digital indicators without any room for flexibility. Taking the output of academic achievements as an example, it is commonplace and generally accepted objective fact that the number of published papers is clearly regarded as a mandatory requirement for graduate students, especially for doctoral graduates, using the number of papers, class hours, and project funding as the rigid requirements for the evaluation of teacher professional titles. Evaluation in other senses is also homogeneous, so it won't be explained more here.

\subsection{Digital discipline: Irrational "digit" becomes disciplinary force by alienation in higher education evaluation}

The rational use of numbers in higher education evaluation must be based on the objective analysis and scientific understanding of numbers, which can effectively mobilize the enthusiasm of the evaluation objects and guide the evaluation objects to achieve self-organized operation that conform to the connotative development of higher education. However, once this evaluative digit has irrational characteristics and becomes a ubiquitous force, the institutional choice of education in the higher education field and the micro-structure of school education are transferred from digital education evaluation, and various educational behaviors in reality take it as a kind of guidance. Then, higher education evaluation would become a new objective force characterized by numbers. [3]At the same time, with the large-scale and high-frequency application of digital scale in higher education evaluation, an all-round and refined evaluation system has been formed around the evaluation objects, and then digital evaluation would become a discipline technique for controlling evaluation objects. And irrational numbers would ignore the "freedom," "openness," and "creation" in the soul of higher education and become distorted into disciplinary force. This kind of external disciplinary force is so great, which is prominently reflected in the "digital supremacy" in higher education field. At this time, the complex evaluation of higher education has been reduced to a simplistic counting task, "winning by numbers" has become the first survival rule, 
and digital indicators have become the behavioral principles and ultimate pursuits to guide the evaluation objects. In this process, the human character and social conscience of higher education have been neglected intentionally or unintentionally. For example, in the evaluation of disciplines, in order to stick to and seize the national rankings of so-called dominant disciplines, schools at all levels use the name of "integrated resources" to start digital games, dismantling non-key, non-characteristic, and non-dominant disciplines as the price, for ensuring a stable ranking of subjects in the central area of the Matthew Effect. It should be noted that the appraisal object gradually loses discernment under the hold of the "digital goal", and abandons the spiritual character and social responsibility of higher education, pilling up and manufacturing various numbers. For example, the current academic misconduct in the field of colleges and universities has no doubt to be the consequence of some university teachers taking risks to achieve the digital goal of assessment under the pressure of huge scientific research.

\section{Probe into the Causes of "Digital Dependence" in Higher}

\section{Education Evaluation}

Digital dependence in higher education evaluation is essentially taking the evaluation scale carried by "number" as the fundamental scale of higher education evaluation and transforming it from an instrumental existence to a purposeful existence, which is a distortion of the digital scale in higher education evaluation. Probe into its root causes, digital dependence is the result of the convergence and interweaving of multiple factors such as technicalism, reductionism, and performanceism.

\subsection{Long-term restriction by the evaluation views on rationalism under the control of technological rationality}

Looking back on the development process of higher education digital evaluation under the dominance of the rationalist evaluation concept, it was initially embodied in the quantification of traditional examination results, and then developed into the educational measurement under the control of scientism. And after that the modern educational evaluation method represented by Taylor's eight years' study has become a milestone. Since then, under the promotion of modern education evaluation technology, higher education evaluation has gradually increased the objectivity and precision of evaluation scales, and further strengthened the digital scale. Especially with the development of big data technology since the third scientific and technological revolution, the "digital survival" described by Negroponte has further promoted the penetration of digital scale in the actual higher education evaluation practice. "'Digital Survival' not only depicts a world dominated by the numbers 0 and 1 , but also reveals a change in thinking mode and establishes a new way of life. The whole society seems to have reached a consensus: It is not science to describe social events without scientific quantification, and it is not enough to describe problems correctly without empirical methods." [4]As Shulman said, "quantitative methods have almost become a worldview."[5]. It can be said that the early emphasis on the digital scale in higher education evaluation emerges as the evaluation concept of "digital attachment" and runs through the practice of higher education evaluation, through the extraction of the field in the era of the third wave of science and technology of mankind. So far, a rationalist evaluation view with the

Characteristics of "digital dependence" has been put in an unassailable position in the field of higher education.

\subsection{Promotion of simplifying perception of the higher education field}

The silence layer of the higher education field is obscured and retreated out of the vision of higher education evaluation to a large extent contributed to the principle of digital quantification in higher education evaluation, supporting the legitimacy of the digital scale and making it rule the field. If the rationalist evaluation view confirms the legitimacy of the digital scale from the perspective of evaluation notion, then the veil of the silence layer in higher education field confirms the rationality of the digital scale from the perspective of evaluation object. Therefore, in the practice of higher education evaluation, only the measurable outer layers of higher education are often concerned, and other evaluation standards are constantly being crushed by strengthening the objectivity, accuracy, and rigor of digital scales. At 
this time, the legal status of the digital scale has been further consolidated and strengthened, and it has obtained the absolute authority and priority of sacred inviolability and is widely used in the evaluation of higher education. To a considerable extent, the simplistic cognition of the complex system of higher education, or more precisely the veil of the evaluation objects in the silent layer of higher education field, is a root of profound understandings that leads to the current digital dependence problem in higher education evaluation.

\subsection{Resource black hole and benefit inducement that "digit" can be manifested}

Examining the theoretical assumptions behind the evaluation of higher education, performances occupies an important position, and it subtly affects the thoughts and behaviors of the subject and object of the evaluation. Performances "centers on performance, focuses on the decomposition of indicators and tasks, uses refined quantitative assessment as leverage, and uses economic rewards and punishments as basic means." [6]Its fundamental value orientation lies in quantitatively evaluating performance and it compares evaluation results with rewards and punishments closely linked in order to encourage evaluation objects. It is precisely under this performances orientation that higher education evaluation places great emphasis on numerical indicators, ranking of results, etc., and it highly links the evaluation results marked by numbers with the allocation of resource. At this time, the digital scale of higher education evaluation and the allocation of resource constitute an "external and internal" relationship. The evaluation of higher education shows a high degree of dependence on numbers. This is only an external appearance, but its essence is the resource allocation mechanism rooted in numbers.

\section{Ways of Action to Dissolve the "Digital Dependence" of Higher Education Evaluation}

\subsection{Returning the meaning of digital criterion in higher education evaluation to be genuine}

The digital dependence in higher education evaluation is essentially the purpose of digital alienation as an evaluation tool. Therefore, governing the problem of digital dependence could not mechanically eliminate numbers in the evaluation of higher education. The rational strategy should first be to bring the alienated numbers back to the origins. That is to establish a meaningful connection point between numbers and the quality of higher education among the rational choices of numbers, so that numbers can truly reflect the quality of higher education and become meaningful evaluation symbols.

\subsection{Clarifying the reasonable boundaries of the digital application in the evaluation of higher education}

Each aspect of the educational field has its own scale for displaying its nature, and numbers cannot be universally applied to the overall time and space of the higher education field. The single linearity, superficiality, and confirmation of numbers are inevitably incompatible with the richness, depth and difference of the quality of higher education, which determines the boundaries of the digit application in the evaluation of higher education. When digit exceeds its own application boundary, the evaluation of higher education shows its corresponding digital dependence. For this reason, the evaluation of higher education must take full account of the complexity of higher education, use numbers rationally, and confirm the reasonable boundaries of the digit application.

\subsection{Optimizing the structure of the higher education evaluation system}

Higher education evaluation is a complex and systematic project. Scattered and superficial reforms could surely help eliminate digital dependence to a certain extent. Such as the representative work system in the evaluation of professional titles, and the digital tendency to dilute the evaluation indicators of various projects. However, the above measures are still trivial explorations and attempts to overcome the digital dependence in higher education evaluation. Therefore, in-depth higher education evaluation and overall optimization of the evaluation structure in higher education should be the direction of further efforts to fundamentally solve this persistent problem. 


\section{Conclusion}

Utilitarian value orientation has been questioned in the evaluation of higher education. Its wide scope, deep degree, and great influence have aroused theoretical deliberations by scholars and a great concern from the central government. Governing this disease has become the fundamental policy orientation for higher education evaluation reform in the new era.

Extremely one-sided, simplified, and rigid evaluation. The most intuitive manifestation it has is the supremacy of numbers and the flooding of meaningless numbers in higher education evaluation, which directly contributes to the digital dependence of utilitarian value orientation. It is true that in the dual environment of the advent of the big data era and the immature qualitative evaluation mechanism, numbers, as an indispensable evaluation tool, should play an irreplaceable role in the evaluation of higher education. However, when digit, which is an evaluation tool, becomes the dominant evaluation scale by alienation and falls into an "ideology" with a "disciplinary" nature, promoting digital dependence, and when overwhelming digital dependence indulges the utilitarian value, it is a practical measure for evaluation of higher education in the new era to reexamine and respond the digital scale and to find solutions to break the utilitarian value orientation. For clarifying the above problems, this research proposes the concept of "digital dependence" to characterize the excessive dependence of higher education evaluation on digital tools and the resulting disciplinary malaise it makes, and also analyzes the actual representation and roots of causes of digital dependence in higher education evaluation. And then this research put forward the solutions to dispel digital dependence from the dimensions of digital tools application and optimization of the higher education evaluation system. However, the above-mentioned measures are still "partial governance" based on the numbers themselves, evaluation methods, and evaluation indicators. It should be noted that the evaluation problem in higher education mapped by digital dependence is quite stubborn and complex. Solving this problem should not only involve in-depth inquiry into the pursuit of genuine value in higher education evaluation, but also require higher education evaluation ecology and even the whole systematic changes in higher education management. It is the key and difficult point for evaluation reform of higher education in the new era to strengthen the professionalism and credibility of qualitative evaluations such as peer evaluation and representative work system, to reshape the social trust of professional authority in higher education evaluation, to empower higher education evaluation with big data technology and intelligent technology, and to change the management thinking and logic of universities. And there is still a lot of room for further research.

\section{REFERENCES}

[1] The Central Committee of the Communist Party of China and the State Council issued the General Plan for Deepening the Reform of Education Evaluation in the New Era.[EB/OL]. (2020-10-13) [2021-06-01]. http://www.gov.cn.

[2] Wang C.L. [The Phenomenon of Number Worship in the Process of Popularization of Higher Education]. [J]. Higher Education of Jiangsu. 2008(02):38-40. (in Chinese)

[3] Jin S.H. [The Disciplinary Concerns of Big Data Educational Evaluation: A Philosophical Review of Educational Instrumentalization]. [J]. Education Research. 2019(08):33-41. (in Chinese)

[4] Cai L.Q.[The inundation and correction of quantitative Evaluation of college students]. [J]. Qingnian Research of China. 2009(08): 97-99.(in Chinese)

[5] E. Shulman.Netherlands.The age of science and technology and the future of mankind: challenges in the depth of philosophy [M]. Translated by:Li X.B. et al. Beijing: Dongfang Press, 1995: 345.(in Chinese)

[6] Yang X.F.,He W.H.[The impact of new managementism on public education reform and its reflection]. [J]. Education Development Research. 2013(10): 73-77. (in Chinese)

[7] Yao J.L.[The lack and development of college management wisdom from the perspective of tacit knowledge].[J].University Education Management, 2016(06): 21-26. (in Chinese)

[8] Liu S.X.["Digital Trap" in Higher Education Evaluation]. [J]. Journal of Suzhou University (Educational Science Edition), 2016(01): 28-35.(in Chinese) 